\title{
LIKELIHOOD RATIO TEST FOR MEAN VECTOR WITH SPECIFIED DIRECTION BASED ON CONDITIONAL DISTRIBUTION
}

\author{
Tsunehisa Imada* and Hideyuki Douke ${ }^{\dagger}$
}

\begin{abstract}
The aim of this study is to construct simple methods of a multivariate one-sided test and a multivariate two-sided test when the covariance matrix is unknown. First we assume that the mean vector has a specified direction as Tang, Gnecco and Geller (1989) considered. Then we set up two kinds of composite hypotheses and derive the likelihood ratio test statistic for our composite hypotheses. However the explicit formula of distribution of this statistic does not exist. Thus we use the conditional distribution considered by Wang and McDermott (1998) to determine the critical value independently of the unknown covariance matrix. Finally we compare our methods with the method of Wang and McDermott (1998) in terms of a numerical example regarding the power of the test.
\end{abstract}

\section{Introduction}

A multivariate one-sided test and a multivariate two-sided test are used for testing the superiority and the inferiority between two treatments evaluated by multivariate normal response. Furthermore a multivariate one-sided test is applied to the multiple comparison for several means with ordered restrictions. Originally in a multivariate one-sided test we assume that the mean vector consists of non-negative components and we test whether the vector is equal to zero or not. Kudo (1963) derived the likelihood ratio test statistic for this one-sided test and determined the distribution of this statistic when the covariance matrix is known. Furthermore Perlman (1969) derived the likelihood ratio test statistic when the covariance matrix is unknown. But in Perlman's procedure the null distribution of the likelihood ratio test statistic depends on the unknown covariance matrix, so it is accompanied by some problems with the power of the test, because the upper and lower bounds of the null distribution are used for determining the critical value and the power of the test. Thus Wang and McDermott (1998) used the conditional distribution given the sufficient statistic for the covariance matrix in order to determine the critical value independently of the unknown covariance matrix. Although the composite hypotheses considered in these studies are suitable for practical use, the procedures derived by likelihood ratio method are theoretically complicated. Concretely the likelihood ratio test statistic is determined by the orthogonal projection of the observed point onto the region of the null hypothesis, so the formulation of the statistic varies with the region to which the observed point belongs

\footnotetext{
*School of Engineering, Kyushu Tokai University, 9-1-1, Toroku, Kumamoto 862-8652, Japan E-mail: timada@ktmail.ktokai-u.ac.jp

†School of Science, Tokai University, 1117, Kitakaname, Hiratsuka, Kanagawa 259-1292, Japan

Key words: Multivariate one-sided test; Multivariate two-sided test; Unknown covariance matrix
} 
and it is difficult to determine the critical value for a specified significance level when the number of variables is large. On the other hand Sasabuchi (1980) derived a one-sided test and a two-sided test based on different composite hypotheses when the covariance matrix is known. Especially in this one-sided test the critical value for a specified significance level is easily determined by using the univariate normal distribution. Furthermore Sasabuchi (1988) developed the procedures when the covariance matrix is unknown. On the other hand Tang, Gnecco and Geller (1989) constructed a simple method of a multivariate onesided test by assuming that the mean vector has a specified direction when the covariance matrix is known. In this method the statistic derived by the likelihood ratio method is distributed according to the univariate normal distribution. Although it is occasionally difficult to decide this direction and the specified direction may be different from the true direction, the method is more powerful than the method of Kudo (1963) when the specified direction is consistent with the true direction.

In the bivariate case with known covariance matrix Kudo and Fujisawa (1964) proposed a two-sided test with composite hypotheses and Inada (1978a, 1978b) proposed various types of one-sided tests and two-sided tests. Furthermore Imada and Douke (2000) developed Kudo-Fujisawa's procedure when the covariance matrix is unknown.

In this study we propose simple methods of a multivariate one-sided test and a multivariate two-sided test when the covariance matrix is unknown. First we assume that the mean vector has a specified direction as Tang et al. (1989) considered. Then we set up two kinds of composite hypotheses and derive the likelihood ratio test statistic for these hypotheses. However the explicit formula of the distribution of this statistic does not exist. Thus we construct the acceptance region and the rejection region based on the statistic and determine the critical value and the power of the test by multiple integration on these regions. Here we use the conditional distribution considered by Wang and McDermott (1998) to determine the critical value independently of the unknown covariance matrix. Finally we compare our methods with the method of Wang and McDermott (1998) in terms of a numerical example regarding the power of the test.

\section{One-sided test and two-sided test based on multivariate normal response}

We suppose that $p$-dimensional random variable vector $\boldsymbol{X}$ is distributed according to $p$-dimensional normal $N(\boldsymbol{\mu}, \boldsymbol{\Sigma})$ with mean vector $\boldsymbol{\mu}$ and unknown covariance matrix $\boldsymbol{\Sigma}$. We assume

$$
\boldsymbol{\mu}=\lambda \delta
$$

where $\lambda$ is an unknown scalar and

$$
\boldsymbol{\delta}=\left(\delta_{1}, \delta_{2}, \ldots, \delta_{p}\right)^{\prime}
$$

is a known $p$-dimensional vector satisfying

$$
\delta_{1} \geq 0, \delta_{2} \geq 0, \ldots, \delta_{p} \geq 0 \quad\left(\delta_{i}>0 \text { for some } i\right) .
$$

Here we consider two kinds of tests as follows:

$$
\begin{array}{ll}
\text { O-S } & H_{0}: \lambda=0 \text { versus } H_{1}: \lambda>0, \\
\text { T-S } & H_{0}: \lambda=0 \text { versus } H_{1}: \lambda \neq 0 .
\end{array}
$$

O-S is a one-sided test and T-S is a two-sided test. Tang et al. (1989) derived the likelihood ratio test for $\mathbf{O}-\mathbf{S}$ when the covariance matrix is known. We construct the likelihood ratio test for $\mathbf{O}-\mathbf{S}$ and $\mathbf{T}-\mathbf{S}$ under the assumption that the covariance matrix is unknown. 


\section{Likelihood ratio method}

We derive the likelihood ratio test statistic for our composite hypotheses. Let

$$
\boldsymbol{X}_{1}=\left(X_{11}, \ldots, X_{1 p}\right)^{\prime}, \boldsymbol{X}_{2}=\left(X_{21}, \ldots, X_{2 p}\right)^{\prime}, \ldots, \boldsymbol{X}_{n}=\left(X_{n 1}, \ldots, X_{n p}\right)^{\prime}
$$

be a sample from $N(\lambda \boldsymbol{\delta}, \boldsymbol{\Sigma})$. Then the likelihood function is

$$
\begin{aligned}
& L=\prod_{i=1}^{n} \frac{1}{(2 \pi)^{\frac{p}{2}}|\Sigma|^{\frac{1}{2}}} \exp \left[-\frac{1}{2}\left(\boldsymbol{X}_{i}-\lambda \delta\right)^{\prime} \boldsymbol{\Sigma}^{-1}\left(\boldsymbol{X}_{i}-\lambda \boldsymbol{\delta}\right)\right] \\
&=\frac{1}{(2 \pi)^{\frac{n p}{2}}|\Sigma|^{\frac{n}{2}}} \exp \left[-\frac{1}{2} \sum_{i=1}^{n}\left(\boldsymbol{X}_{i}-\lambda \boldsymbol{\delta}\right)^{\prime} \boldsymbol{\Sigma}^{-1}\left(\boldsymbol{X}_{i}-\lambda \boldsymbol{\delta}\right)\right] \\
&=\frac{1}{(2 \pi)^{\frac{n p}{2}}|\Sigma|^{\frac{n}{2}}} \exp \left[-\frac{1}{2}\left\{\sum_{i=1}^{n}\left(\boldsymbol{X}_{i}-\overline{\boldsymbol{X}}\right)^{\prime} \boldsymbol{\Sigma}^{-1}\left(\boldsymbol{X}_{i}-\overline{\boldsymbol{X}}\right)\right.\right. \\
&\left.\left.+n(\overline{\boldsymbol{X}}-\lambda \boldsymbol{\delta})^{\prime} \boldsymbol{\Sigma}^{-1}(\overline{\boldsymbol{X}}-\lambda \boldsymbol{\delta})\right\}\right]
\end{aligned}
$$

where $\overline{\boldsymbol{X}}=\left(\bar{X}_{1}, \ldots, \bar{X}_{p}\right)^{\prime}=\frac{1}{n} \sum_{i=1}^{n} \boldsymbol{X}_{i}$. We derive the maximum likelihood $L_{0}$ and $L_{1}$ under $H_{0}$ and $H_{0} \cup H_{1}$ respectively.

First we fix the value of $\lambda$ and determine the value of $\boldsymbol{\Sigma}$ which maximizes $L$. In (4)

$$
\begin{aligned}
& \sum_{i=1}^{n}\left(\boldsymbol{X}_{i}-\overline{\boldsymbol{X}}\right)^{\prime} \boldsymbol{\Sigma}^{-1}\left(\boldsymbol{X}_{i}-\overline{\boldsymbol{X}}\right)+n(\overline{\boldsymbol{X}}-\lambda \boldsymbol{\delta})^{\prime} \boldsymbol{\Sigma}^{-1}(\overline{\boldsymbol{X}}-\lambda \boldsymbol{\delta}) \\
& =\operatorname{tr} \boldsymbol{\Sigma}^{-1}\left\{\sum_{i=1}^{n}\left(\boldsymbol{X}_{i}-\overline{\boldsymbol{X}}\right)\left(\boldsymbol{X}_{i}-\overline{\boldsymbol{X}}\right)^{\prime}+n(\overline{\boldsymbol{X}}-\lambda \boldsymbol{\delta})(\overline{\boldsymbol{X}}-\lambda \boldsymbol{\delta})^{\prime}\right\} \\
& =\operatorname{tr} \boldsymbol{\Sigma}^{-1}\left\{\boldsymbol{S}+n(\overline{\boldsymbol{X}}-\lambda \boldsymbol{\delta})(\overline{\boldsymbol{X}}-\lambda \boldsymbol{\delta})^{\prime}\right\}
\end{aligned}
$$

where

$$
\boldsymbol{S}=\sum_{i=1}^{n}\left(\boldsymbol{X}_{i}-\overline{\boldsymbol{X}}\right)\left(\boldsymbol{X}_{i}-\overline{\boldsymbol{X}}\right)^{\prime}
$$

Thus

$$
\log L=-\frac{n p}{2} \log 2 \pi-\frac{n}{2} \log |\boldsymbol{\Sigma}|-\frac{1}{2} \operatorname{tr} \boldsymbol{\Sigma}^{-1}\left\{\boldsymbol{S}+n(\overline{\boldsymbol{X}}-\lambda \boldsymbol{\delta})(\overline{\boldsymbol{X}}-\lambda \boldsymbol{\delta})^{\prime}\right\} .
$$

By Anderson (1984), Lemma 3.2.2, $L$ is maximized when

$$
\boldsymbol{\Sigma}=\frac{1}{n}\left\{\boldsymbol{S}+n(\overline{\boldsymbol{X}}-\lambda \boldsymbol{\delta})(\overline{\boldsymbol{X}}-\lambda \boldsymbol{\delta})^{\prime}\right\}
$$

By substituting (6) into (5)

$$
\log L=-\frac{n p}{2}(\log 2 \pi+1)-\frac{n}{2} \log \left|\frac{1}{n}\left\{\boldsymbol{S}+n(\overline{\boldsymbol{X}}-\lambda \boldsymbol{\delta})(\overline{\boldsymbol{X}}-\lambda \boldsymbol{\delta})^{\prime}\right\}\right| .
$$

Furthermore by Anderson (1984), Corollary A.3.1,

$$
\left|\boldsymbol{S}+n(\overline{\boldsymbol{X}}-\lambda \boldsymbol{\delta})(\overline{\boldsymbol{X}}-\lambda \boldsymbol{\delta})^{\prime}\right|=|\boldsymbol{S}|\left\{1+n(\overline{\boldsymbol{X}}-\lambda \boldsymbol{\delta})^{\prime} \boldsymbol{S}^{-1}(\overline{\boldsymbol{X}}-\lambda \boldsymbol{\delta})\right\} .
$$

Thus

$$
\log L=-\frac{n p}{2}(\log 2 \pi+1)-\frac{n}{2} \log \left|\frac{\boldsymbol{S}}{n}\right|-\frac{n}{2} \log \left\{1+n(\overline{\boldsymbol{X}}-\lambda \boldsymbol{\delta})^{\prime} \boldsymbol{S}^{-1}(\overline{\boldsymbol{X}}-\lambda \boldsymbol{\delta})\right\} .
$$


We denote the maximum likelihood estimator of $\lambda$ by $\hat{\lambda}$. Since $\hat{\lambda}=0$ under $H_{0}: \lambda=0$,

$$
\log L_{0}=-\frac{n p}{2}(\log 2 \pi+1)-\frac{n}{2} \log \left|\frac{\boldsymbol{S}}{n}\right|-\frac{n}{2} \log \left(1+n \overline{\boldsymbol{X}}^{\prime} \boldsymbol{S}^{-1} \overline{\boldsymbol{X}}\right) .
$$

On the other hand by (8) $\hat{\lambda}$ under $H_{0} \cup H_{1}$ is the value of $\lambda$ minimizing

$$
(\overline{\boldsymbol{X}}-\lambda \boldsymbol{\delta})^{\prime} \boldsymbol{S}^{-1}(\overline{\boldsymbol{X}}-\lambda \boldsymbol{\delta}) .
$$

Since

$$
(\overline{\boldsymbol{X}}-\lambda \boldsymbol{\delta})^{\prime} \boldsymbol{S}^{-1}(\overline{\boldsymbol{X}}-\lambda \boldsymbol{\delta})=\boldsymbol{\delta}^{\prime} \boldsymbol{S}^{-1} \boldsymbol{\delta}\left(\lambda-\frac{\boldsymbol{\delta}^{\prime} \boldsymbol{S}^{-1} \overline{\boldsymbol{X}}}{\boldsymbol{\delta}^{\prime} \boldsymbol{S}^{-1} \boldsymbol{\delta}}\right)^{2}+\overline{\boldsymbol{X}}^{\prime} \boldsymbol{S}^{-1} \overline{\boldsymbol{X}}-\frac{\left(\boldsymbol{\delta}^{\prime} \boldsymbol{S}^{-1} \overline{\boldsymbol{X}}\right)^{2}}{\boldsymbol{\delta}^{\prime} \boldsymbol{S}^{-1} \boldsymbol{\delta}}
$$

$\hat{\lambda}$ under $H_{0} \cup H_{1}$ is determined as follows:

(1) Test O-S

Case 1. When $\boldsymbol{\delta}^{\prime} \boldsymbol{S}^{-1} \overline{\boldsymbol{X}} \geq 0$, then

$$
\hat{\lambda}=\frac{\delta^{\prime} \boldsymbol{S}^{-1} \overline{\boldsymbol{X}}}{\boldsymbol{\delta}^{\prime} \boldsymbol{S}^{-1} \boldsymbol{\delta}}
$$

Case 2. When $\boldsymbol{\delta}^{\prime} \boldsymbol{S}^{-1} \overline{\boldsymbol{X}}<0$, then

$$
\hat{\lambda}=0 .
$$

(2) Test T-S

$$
\hat{\lambda}=\frac{\boldsymbol{\delta}^{\prime} \boldsymbol{S}^{-1} \overline{\boldsymbol{X}}}{\boldsymbol{\delta}^{\prime} \boldsymbol{S}^{-1} \boldsymbol{\delta}}
$$

Thus, when $\hat{\lambda}=\boldsymbol{\delta}^{\prime} \boldsymbol{S}^{-1} \overline{\boldsymbol{X}} / \boldsymbol{\delta}^{\prime} \boldsymbol{S}^{-1} \boldsymbol{\delta}$,

$$
\log L_{1}=-\frac{n p}{2}(\log 2 \pi+1)-\frac{n}{2} \log \left|\frac{\boldsymbol{S}}{n}\right|-\frac{n}{2} \log \left(1+n \overline{\boldsymbol{X}}^{\prime} \boldsymbol{S}^{-1} \overline{\boldsymbol{X}}-n \frac{\left(\boldsymbol{\delta}^{\prime} \boldsymbol{S}^{-1} \overline{\boldsymbol{X}}\right)^{2}}{\boldsymbol{\delta}^{\prime} \boldsymbol{S}^{-1} \boldsymbol{\delta}}\right)
$$

and the likelihood ratio is

$$
\frac{L_{0}}{L_{1}}=\left(1-\frac{\left(\boldsymbol{\delta}^{\prime} \boldsymbol{S}^{-1} \boldsymbol{Z}\right)^{2}}{\boldsymbol{\delta}^{\prime} \boldsymbol{S}^{-1} \boldsymbol{\delta}\left(1+\boldsymbol{Z}^{\prime} \boldsymbol{S}^{-1} \boldsymbol{Z}\right)}\right)^{\frac{n}{2}}
$$

where $\boldsymbol{Z}=\left(Z_{1}, \ldots, Z_{p}\right)^{\prime}=\sqrt{n} \overline{\boldsymbol{X}}$. On the other hand, when $\hat{\lambda}=0$ under $H_{0} \cup H_{1}$,

$$
\frac{L_{0}}{L_{1}}=1
$$

Thus we obtain the likelihood ratio test statistic

$$
\boldsymbol{T}=\frac{\delta^{\prime} \boldsymbol{S}^{-1} \boldsymbol{Z}}{\sqrt{\delta^{\prime} S^{-1} \delta\left(1+Z^{\prime} S^{-1} Z\right)}}
$$

and the rejection region of $H_{0}$ for a critical value $c$ is given as follows:

$$
\begin{array}{ll}
\boldsymbol{T}>c & \text { for } \mathbf{O}-\mathbf{S} \\
|\boldsymbol{T}|>c & \text { for } \mathbf{T}-\mathbf{S} .
\end{array}
$$




\section{Determination of critical value by conditional distribution}

If we specify a critical value $c$, the probability that $H_{0}$ is rejected is

$$
P(\boldsymbol{T}>c) \quad \text { for } \mathbf{O}-\mathbf{S}
$$

and

$$
P(|\boldsymbol{T}|>c) \quad \text { for } \mathbf{T}-\mathbf{S} .
$$

The critical value $c$ should be chosen so that these probabilities under $H_{0}$ may be equal to a specified significance level $\alpha$. But the explicit formula of the distribution of $\boldsymbol{T}$ does not exist. So we have to determine $c$ by a different way. We define a set $\boldsymbol{D}(c)\left(\subset \boldsymbol{R}^{p}\right)$ as

$$
\boldsymbol{D}(c)=\left\{\boldsymbol{z} \in \boldsymbol{R}^{p}: \boldsymbol{T}>c\right\} \quad \text { for } \mathbf{O}-\mathbf{S}
$$

and

$$
\boldsymbol{D}(c)=\left\{\boldsymbol{z} \in \boldsymbol{R}^{p}:|\boldsymbol{T}|>c\right\} \quad \text { for T-S. }
$$

Then $\boldsymbol{D}(c)$ is the rejection region of $H_{0}$. Thus the probability that $H_{0}$ is rejected under $H_{0}$ is equal to

$$
\int \cdots \int_{\boldsymbol{D}(c)} f_{0}(\boldsymbol{z}, \boldsymbol{\Sigma}) d \boldsymbol{z}
$$

where $f_{0}(\boldsymbol{z}, \boldsymbol{\Sigma})$ is the probability density function of $N(\mathbf{0}, \boldsymbol{\Sigma})$ and $d \boldsymbol{z}=d z_{1} d z_{2} \cdots d z_{p}$. But (13) depends on the unknown $\boldsymbol{\Sigma}$. Therefore we should determine the critical value $c$ so that the supremum of the multiple integration in (13) for all $\boldsymbol{\Sigma}$

$$
\sup _{\boldsymbol{\Sigma}} \int \cdots \int_{\boldsymbol{D}(c)} f_{0}(\boldsymbol{z}, \boldsymbol{\Sigma}) d \boldsymbol{z}
$$

may be equal to $\alpha$. But we cannot determine this supremum. Thus we use the conditional distribution considered by Wang and McDermott (1998).

\subsection{Conditional distribution}

Since

$$
\boldsymbol{V}=\left(V_{i j}\right)=\sum_{i=1}^{n} \boldsymbol{X}_{i} \boldsymbol{X}_{i}^{\prime}
$$

is the complete and sufficient statistic for $\boldsymbol{\Sigma}$ under $H_{0}$, the conditional distribution of $\boldsymbol{Z}$ given $\boldsymbol{V}$ does not depend on $\boldsymbol{\Sigma}$. Thus by this conditional distribution, the probability that $H_{0}$ is rejected can be calculated independently of $\boldsymbol{\Sigma}$. Thus we have to derive the density function of this conditional distribution. $\boldsymbol{S}$ and $\boldsymbol{Z}$ are independently distributed with

$$
\boldsymbol{S} \sim W(\boldsymbol{\Sigma}, n-1)
$$

and

$$
\boldsymbol{Z} \sim N(\mathbf{0}, \boldsymbol{\Sigma})
$$

under $H_{0}$ where $W(\boldsymbol{\Sigma}, n-1)$ is the Wishart distribution. Thus if $s$ is positive definite (hereafter denoted by $\boldsymbol{s}>0$ ), the joint density of $\boldsymbol{S}$ and $\boldsymbol{Z}$ is

$$
f_{0}(\boldsymbol{z}, \boldsymbol{s})=k_{0}|\boldsymbol{\Sigma}|^{-\frac{1}{2}} \exp \left(-\frac{1}{2} z^{\prime} \boldsymbol{\Sigma}^{-1} \boldsymbol{z}\right) \times|\boldsymbol{\Sigma}|^{-\frac{n-1}{2}}|s|^{\frac{n-p-2}{2}} \exp \left(-\frac{1}{2} \operatorname{tr} \boldsymbol{\Sigma}^{-1} \boldsymbol{s}\right)
$$


where $k_{0}$ is a constant. Since $\boldsymbol{z}^{\prime} \boldsymbol{\Sigma}^{-1} \boldsymbol{z}=\operatorname{tr} \boldsymbol{\Sigma}^{-1} \boldsymbol{z} \boldsymbol{z}^{\prime}$,

$$
f_{0}(\boldsymbol{z}, \boldsymbol{s})=k_{0}|\boldsymbol{\Sigma}|^{-\frac{n}{2}}|\boldsymbol{s}|^{\frac{n-p-2}{2}} \exp \left\{-\frac{1}{2} \operatorname{tr} \boldsymbol{\Sigma}^{-1}\left(\boldsymbol{s}+\boldsymbol{z} \boldsymbol{z}^{\prime}\right)\right\} .
$$

On the other hand if $s$ is not positive definite, $f_{0}(z, s)=0$. Since

$$
s=\sum_{i=1}^{n}\left(\boldsymbol{x}_{i}-\overline{\boldsymbol{x}}\right)\left(\boldsymbol{x}_{i}-\overline{\boldsymbol{x}}\right)^{\prime}=\sum_{i=1}^{n} \boldsymbol{x}_{i} \boldsymbol{x}_{i}^{\prime}-n \overline{\boldsymbol{x}} \overline{\boldsymbol{x}}^{\prime}=\boldsymbol{v}-\boldsymbol{z} \boldsymbol{z}^{\prime},
$$

the Jacobian of the transformation from $(\boldsymbol{z}, \boldsymbol{s})$ to $(\boldsymbol{z}, \boldsymbol{v})$ is 1 . By (15) and (16) the joint density of $\boldsymbol{V}$ and $\boldsymbol{Z}$ is

$$
g_{0}(\boldsymbol{z}, \boldsymbol{v})= \begin{cases}k_{0}|\boldsymbol{\Sigma}|^{-\frac{n}{2}}\left|\boldsymbol{v}-\boldsymbol{z} \boldsymbol{z}^{\prime}\right|^{\frac{n-p-2}{2}} \exp \left(-\frac{1}{2} \operatorname{tr} \boldsymbol{\Sigma}^{-1} \boldsymbol{v}\right) & \text { if } \boldsymbol{v}-\boldsymbol{z} \boldsymbol{z}^{\prime}>0 \\ 0 & \text { otherwise. }\end{cases}
$$

We define $p(\boldsymbol{z}, \boldsymbol{v})$ by

$$
p(\boldsymbol{z}, \boldsymbol{v})= \begin{cases}1 & \text { if } \boldsymbol{v}-\boldsymbol{z z ^ { \prime }}>0 \\ 0 & \text { otherwise. }\end{cases}
$$

Then we can express $g_{0}(\boldsymbol{z}, \boldsymbol{v})$ uniquely as

$$
g_{0}(\boldsymbol{z}, \boldsymbol{v})=k_{0}|\boldsymbol{\Sigma}|^{-\frac{n}{2}}\left|\boldsymbol{v}-\boldsymbol{z} \boldsymbol{z}^{\prime}\right|^{\frac{n-p-2}{2}} \exp \left(-\frac{1}{2} \operatorname{tr} \boldsymbol{\Sigma}^{-1} \boldsymbol{v}\right) p(\boldsymbol{z}, \boldsymbol{v}) .
$$

Since

we obtain

$$
z_{i}^{2}=\frac{1}{n}\left(\sum_{k=1}^{n} x_{k i}\right)^{2} \leq \sum_{k=1}^{n} x_{k i}^{2}=v_{i i}
$$

Thus the marginal density of $\boldsymbol{V}$ is

$$
-\sqrt{v_{i i}} \leq z_{i} \leq \sqrt{v_{i i}}
$$

$$
h_{0}(\boldsymbol{v})=k_{0}|\boldsymbol{\Sigma}|^{-\frac{n}{2}} \exp \left(-\frac{1}{2} \operatorname{tr} \boldsymbol{\Sigma}^{-1} \boldsymbol{v}\right) l_{0}(\boldsymbol{v})
$$

where

$$
l_{0}(\boldsymbol{v})=\int_{-\sqrt{v_{p p}}}^{\sqrt{v_{p p}}} \cdots \int_{-\sqrt{v_{11}}}^{\sqrt{v_{11}}}\left|\boldsymbol{v}-\boldsymbol{z} \boldsymbol{z}^{\prime}\right|^{\frac{n-p-2}{2}} p(\boldsymbol{z}, \boldsymbol{v}) d \boldsymbol{z} .
$$

By (18) and (19) the conditional density of $\boldsymbol{Z}$ given $\boldsymbol{V}=\boldsymbol{v}$ is

$$
g_{0}(\boldsymbol{z} \mid \boldsymbol{v})=k\left|\boldsymbol{v}-\boldsymbol{z} \boldsymbol{z}^{\prime}\right|^{\frac{n-p-2}{2}} p(\boldsymbol{z}, \boldsymbol{v})
$$

where $k$ is a constant. We define a set $\boldsymbol{\Omega}\left(\subset \boldsymbol{R}^{p}\right)$ and a function $I_{\boldsymbol{\Omega}}(\boldsymbol{z})$ on $\boldsymbol{R}^{p}$ as

$$
\boldsymbol{\Omega}=\left\{\boldsymbol{z} \in \boldsymbol{R}^{p}: \boldsymbol{v}-\boldsymbol{z} \boldsymbol{z}^{\prime}>0,-\sqrt{v_{i i}} \leq z_{i} \leq \sqrt{v_{i i}}(i=1,2, \ldots, p)\right\}
$$

and

$$
I_{\boldsymbol{\Omega}}(z)= \begin{cases}1 & \text { if } z \in \boldsymbol{\Omega} \\ 0 & \text { if } z \notin \boldsymbol{\Omega}\end{cases}
$$

By Seber (1977), $v-z z^{\prime}>0$ is equivalent to the condition that all of the leading minor determinants of $\boldsymbol{v}-\boldsymbol{z} \boldsymbol{z}^{\prime}$ and the determinant of $\boldsymbol{v}-\boldsymbol{z} \boldsymbol{z}^{\prime}$ are positive. Since $I_{\boldsymbol{\Omega}}(\boldsymbol{z})=p(\boldsymbol{z}, \boldsymbol{v})$ for given $v$,

$$
g_{0}(z \mid v)=k\left|v-z z^{\prime}\right|^{\frac{n-p-2}{2}} I_{\boldsymbol{\Omega}}(z) .
$$

Thus $k$ is determined by

$$
k=\left(\int \cdots \int_{\boldsymbol{\Omega}}\left|\boldsymbol{v}-\boldsymbol{z} \boldsymbol{z}^{\prime}\right|^{\frac{n-p-2}{2}} d \boldsymbol{z}\right)^{-1} .
$$




\subsection{Determination of critical value}

We determine the critical value for a specified significance level $\alpha$ by using the conditional distribution derived in the previous section. Since the critical value depends on the given value $\boldsymbol{v}$ of $\boldsymbol{V}$, we denote the critical value by $c(\boldsymbol{v})$. Thus we denote the rejection region of $H_{0}$ by $\boldsymbol{D}(c(\boldsymbol{v}))$. Then the probability that $H_{0}$ is rejected given $\boldsymbol{V}=\boldsymbol{v}$ under $H_{0}$ is

$$
\int \cdots \int_{\boldsymbol{D}(c(\boldsymbol{v}))} g_{0}(\boldsymbol{z} \mid \boldsymbol{v}) d \boldsymbol{z}=k \int \cdots \int_{\boldsymbol{D}(c(\boldsymbol{v}))}\left|\boldsymbol{v}-\boldsymbol{z} \boldsymbol{z}^{\prime}\right|^{\frac{n-p-2}{2}} I_{\boldsymbol{\Omega}}(\boldsymbol{z}) d \boldsymbol{z} .
$$

The right-hand side of $(22)$ is

$$
k \int \cdots \int_{\boldsymbol{D}(c(\boldsymbol{v})) \cap \boldsymbol{\Omega}}\left|\boldsymbol{v}-\boldsymbol{z z ^ { \prime }}\right|^{\frac{n-p-2}{2}} d \boldsymbol{z} .
$$

Thus we determine the critical value $c(\boldsymbol{v})$ such that (23) may be equal to the specified significance level $\alpha$.

\section{Numerical example}

Suppose $p=2, n=30, \boldsymbol{\delta}=(1,1)^{\prime}$ and observed values of $\overline{\boldsymbol{X}}$ and $\boldsymbol{V}$ are given by the following three cases:

$$
\begin{aligned}
& \text { Case 1. } \overline{\boldsymbol{x}}=\left(\begin{array}{l}
0.22 \\
0.22
\end{array}\right), \quad \boldsymbol{v}=\left(\begin{array}{rr}
29.74 & 17.94 \\
17.94 & 29.64
\end{array}\right), \\
& \text { Case 2. } \overline{\boldsymbol{x}}=\left(\begin{array}{l}
0.35 \\
0.37
\end{array}\right), \quad \boldsymbol{v}=\left(\begin{array}{rr}
30.24 & -8.01 \\
-8.01 & 66.24
\end{array}\right), \\
& \text { Case 3. } \overline{\boldsymbol{x}}=\left(\begin{array}{l}
0.58 \\
0.62
\end{array}\right), \quad \boldsymbol{v}=\left(\begin{array}{rr}
29.34 & 5.28 \\
5.28 & 119.70
\end{array}\right) .
\end{aligned}
$$

In each case the value of statistic $\boldsymbol{T}$ is given as follows:

Case 1. 0.247, Case 2. 0.471, Case 3. 0.641.

Then in each case the critical value $c(\boldsymbol{v})$ satisfying significance level $\alpha=0.05$ is determined as follows:

Case 1. $c(\boldsymbol{v})=0.38$ for $\mathbf{O}-\mathbf{S}, c(\boldsymbol{v})=0.45$ for $\mathbf{T}-\mathbf{S}$,

Case 2. $c(\boldsymbol{v})=0.45$ for $\mathbf{O}-\mathbf{S}, c(\boldsymbol{v})=0.52$ for $\mathbf{T}-\mathbf{S}$,

Case 3. $c(\boldsymbol{v})=0.58$ for $\mathbf{O}-\mathbf{S}, c(\boldsymbol{v})=0.62$ for $\mathbf{T}-\mathbf{S}$.

In Case 1 we accept $H_{0}$ for $\mathbf{O}-\mathbf{S}$ and T-S. In Case 2 we accept $H_{0}$ for T-S and reject $H_{0}$ for $\mathbf{O}-\mathbf{S}$. In Case 3 we reject $H_{0}$ for $\mathbf{O}-\mathbf{S}$ and T-S.

\section{Power of the test}

Next we consider the power of the test. Then we will calculate the power for a specified value $\lambda_{1}$ under $H_{1}$, although we have to consider the power for all $\lambda$ under $H_{1}$. Thus we consider the power under $H_{1}: \lambda=\lambda_{1}$ where $\lambda_{1}>0$ for $\mathbf{O}-\mathbf{S}$ and $\lambda_{1} \neq 0$ for T-S. Since $\boldsymbol{V}$ 
is not sufficient for $\boldsymbol{\Sigma}$ under $H_{1}$, the conditional distribution of $\boldsymbol{Z}$ given $\boldsymbol{V}$ depends on $\boldsymbol{\Sigma}$. Denoting the density of this conditional distribution by $g_{1}(z \mid v)$,

$$
\pi\left(\lambda_{1}, \boldsymbol{v}, \boldsymbol{\Sigma}\right)=\int \cdots \int_{\boldsymbol{D}(c(\boldsymbol{v}))} g_{1}(\boldsymbol{z} \mid \boldsymbol{v}) d \boldsymbol{z}
$$

is the power of the test under $H_{1}$ when the value of $\boldsymbol{V}$ is given by $\boldsymbol{V}=\boldsymbol{v}$ and the covariance matrix is $\boldsymbol{\Sigma}$. On the other hand, since

$$
\left|v_{i j}\right|=\left|\sum_{k=1}^{n} x_{k i} x_{k j}\right| \leq \frac{1}{2} \sum_{k=1}^{n}\left(x_{k i}^{2}+x_{k j}^{2}\right)=\frac{1}{2}\left(v_{i i}+v_{j j}\right) \text { for } i \neq j,
$$

the region $R \boldsymbol{v}$ of $\boldsymbol{v}$ consists of $\boldsymbol{v}=\left(v_{i j}\right)$ satisfying

$$
v_{i i} \geq 0, \quad-\frac{1}{2}\left(v_{i i}+v_{j j}\right) \leq v_{i j} \leq \frac{1}{2}\left(v_{i i}+v_{j j}\right) .
$$

Then

$$
\pi\left(\lambda_{1}, \boldsymbol{\Sigma}\right)=\int \cdots \int_{\boldsymbol{R} \boldsymbol{v}} \pi\left(\lambda_{1}, \boldsymbol{v}, \boldsymbol{\Sigma}\right) h_{1}(\boldsymbol{v}) d \boldsymbol{v}
$$

is the power of the test under $H_{1}$, where $h_{1}(\boldsymbol{v})$ is the density of $\boldsymbol{V}$ under $H_{1}, d \boldsymbol{v}$ is the product of $d v_{i j}$ for all $(i, j)$-component $v_{i j}(i \geq j)$ of $\boldsymbol{v}$. Denoting the joint density of $\boldsymbol{V}$ and $\boldsymbol{Z}$ by $g_{1}(\boldsymbol{z}, \boldsymbol{v}),(25)$ is rewritten by

$$
\pi\left(\lambda_{1}, \boldsymbol{\Sigma}\right)=\int \cdots \int_{R_{\boldsymbol{v}}}\left\{\int \cdots \int_{\boldsymbol{D}(c(\boldsymbol{v}))} g_{1}(\boldsymbol{z}, \boldsymbol{v}) d \boldsymbol{z}\right\} d \boldsymbol{v} .
$$

Thus we have to derive $g_{1}(\boldsymbol{z}, \boldsymbol{v}) . \boldsymbol{S}$ and $\boldsymbol{Z}$ are independently distributed with

$$
\boldsymbol{S} \sim W(\boldsymbol{\Sigma}, n-1)
$$

and

$$
\boldsymbol{Z} \sim N\left(\lambda_{1} \sqrt{n} \boldsymbol{\delta}, \boldsymbol{\Sigma}\right)
$$

under $H_{1}$. Thus if $s>0$, the joint density of $\boldsymbol{S}$ and $\boldsymbol{Z}$ is

$$
\begin{aligned}
f_{1}(\boldsymbol{z}, \boldsymbol{s})= & k_{1}|\boldsymbol{\Sigma}|^{-\frac{1}{2}} \exp \left[-\frac{1}{2}\left(\boldsymbol{z}-\lambda_{1} \sqrt{n} \boldsymbol{\delta}\right)^{\prime} \boldsymbol{\Sigma}^{-1}\left(z-\lambda_{1} \sqrt{n} \boldsymbol{\delta}\right)\right] \\
& \times|\boldsymbol{\Sigma}|^{-\frac{n-1}{2}}|\boldsymbol{s}|^{\frac{n-p-2}{2}} \exp \left(-\frac{1}{2} \operatorname{tr} \boldsymbol{\Sigma}^{-1} \boldsymbol{s}\right)
\end{aligned}
$$

where $k_{1}$ is a constant. Since

$$
\left(\boldsymbol{z}-\lambda_{1} \sqrt{n} \boldsymbol{\delta}\right)^{\prime} \boldsymbol{\Sigma}^{-1}\left(\boldsymbol{z}-\lambda_{1} \sqrt{n} \boldsymbol{\delta}\right)=\operatorname{tr} \boldsymbol{\Sigma}^{-1}\left(z-\lambda_{1} \sqrt{n} \boldsymbol{\delta}\right)\left(\boldsymbol{z}-\lambda_{1} \sqrt{n} \boldsymbol{\delta}\right)^{\prime},
$$

$f_{1}(\boldsymbol{z}, \boldsymbol{s})$ is written as

$$
\begin{aligned}
& f_{1}(\boldsymbol{z}, \boldsymbol{s})=k_{1}|\boldsymbol{\Sigma}|^{-\frac{n}{2}}|\boldsymbol{s}|^{\frac{n-p-2}{2}} \exp \left[-\frac{1}{2} \operatorname{tr} \boldsymbol{\Sigma}^{-1}\left\{\boldsymbol{s}+\left(\boldsymbol{z}-\lambda_{1} \sqrt{n} \boldsymbol{\delta}\right)\left(\boldsymbol{z}-\lambda_{1} \sqrt{n} \boldsymbol{\delta}\right)^{\prime}\right\}\right] \\
& =k_{1}|\boldsymbol{\Sigma}|^{-\frac{n}{2}}|\boldsymbol{s}|^{\frac{n-p-2}{2}} \exp \left[-\frac{1}{2} \operatorname{tr} \boldsymbol{\Sigma}^{-1}\left(\boldsymbol{s}+\boldsymbol{z} \boldsymbol{z}^{\prime}-\lambda_{1} \sqrt{n} \boldsymbol{\delta} \boldsymbol{z}^{\prime}-\lambda_{1} \sqrt{n} \boldsymbol{z} \boldsymbol{\delta}^{\prime}+\lambda_{1}^{2} n \boldsymbol{\delta} \boldsymbol{\delta}^{\prime}\right)\right] \\
& =k_{1}|\boldsymbol{\Sigma}|^{-\frac{n}{2}}|\boldsymbol{s}|^{\frac{n-p-2}{2}} \exp \left[-\frac{1}{2} \operatorname{tr} \boldsymbol{\Sigma}^{-1}\left(\boldsymbol{s}+\boldsymbol{z} \boldsymbol{z}^{\prime}+\lambda_{1}^{2} n \boldsymbol{\delta} \boldsymbol{\delta}^{\prime}\right)\right] \exp \left[\frac{\lambda_{1} \sqrt{n}}{2} \operatorname{tr} \boldsymbol{\Sigma}^{-1}\left(\boldsymbol{\delta} \boldsymbol{z}^{\prime}+\boldsymbol{z} \boldsymbol{\delta}^{\prime}\right)\right] .
\end{aligned}
$$


Here

$$
\operatorname{tr} \boldsymbol{\Sigma}^{-1}\left(\boldsymbol{\delta} z^{\prime}+z \boldsymbol{\delta}^{\prime}\right)=\boldsymbol{z}^{\prime} \boldsymbol{\Sigma}^{-1} \boldsymbol{\delta}+\boldsymbol{\delta}^{\prime} \boldsymbol{\Sigma}^{-1} \boldsymbol{z}=2 \boldsymbol{\delta}^{\prime} \boldsymbol{\Sigma}^{-1} \boldsymbol{z}
$$

because $\boldsymbol{\Sigma}^{-1}$ is symmetric. Thus

$$
f_{1}(\boldsymbol{z}, \boldsymbol{s})=k_{1}|\boldsymbol{\Sigma}|^{-\frac{n}{2}}|\boldsymbol{s}|^{\frac{n-p-2}{2}} \exp \left[-\frac{1}{2} \operatorname{tr} \boldsymbol{\Sigma}^{-1}\left(\boldsymbol{s}+\boldsymbol{z} \boldsymbol{z}^{\prime}+\lambda_{1}^{2} n \boldsymbol{\delta} \boldsymbol{\delta}^{\prime}\right)\right] \exp \left(\lambda_{1} \sqrt{n} \boldsymbol{\delta}^{\prime} \boldsymbol{\Sigma}^{-1} \boldsymbol{z}\right) .
$$

On the other hand, if $s$ is not positive definite, $f_{0}(z, s)=0$. By $(27)$, the joint density of $\boldsymbol{V}$ and $\boldsymbol{Z}$ is expressed uniquely as

$$
g_{1}(\boldsymbol{z}, \boldsymbol{v})=k^{\prime}\left|\boldsymbol{v}-\boldsymbol{z} \boldsymbol{z}^{\prime}\right|^{\frac{n-p-2}{2}} \exp \left(\lambda_{1} \sqrt{n} \boldsymbol{\delta}^{\prime} \boldsymbol{\Sigma}^{-1} \boldsymbol{z}-\frac{1}{2} \operatorname{tr} \boldsymbol{\Sigma}^{-1} \boldsymbol{v}\right) p(\boldsymbol{z}, \boldsymbol{v})
$$

where $p(z, v)$ is defined in (17) and $k^{\prime}$ is a constant determined by

$$
\begin{aligned}
k^{\prime}=\left(\int \cdots \int _ { R \boldsymbol { v } } \left\{\int \cdots \int_{\boldsymbol{D}(c(\boldsymbol{v})) \cap \boldsymbol{\Omega}}\left|\boldsymbol{v}-\boldsymbol{z} \boldsymbol{z}^{\prime}\right|^{\frac{n-p-2}{2}}\right.\right. \\
\left.\left.\times \exp \left(\lambda_{1} \sqrt{n} \boldsymbol{\delta}^{\prime} \boldsymbol{\Sigma}^{-1} \boldsymbol{z}-\frac{1}{2} \operatorname{tr} \boldsymbol{\Sigma}^{-1} \boldsymbol{v}\right) d \boldsymbol{z}\right\} d \boldsymbol{v}\right)^{-1} .
\end{aligned}
$$

We can obtain the power of the test $\pi\left(\lambda_{1}, \boldsymbol{\Sigma}\right)$ in (26) by using $g_{1}(\boldsymbol{z}, \boldsymbol{v})$ in (28).

As we discuss in the next section, it is occasionally difficult to decide the direction $\delta$ and the specified direction $\delta$ may be different from the true direction $\delta_{1}$. When we investigate the power of the test with direction $\boldsymbol{\delta}_{1}$ which is different from the specified direction $\delta$, we use the density obtained by substituting $\boldsymbol{\delta}_{1}$ for $\delta$ in (28).

\section{Power comparisons}

We obtain two methods $\mathbf{O}-\mathbf{S}$ and T-S. In the original one-sided test considered by Kudo et al. the formulation of the likelihood ratio test statistic varies with the region to which the observed point belongs. But in our procedures the likelihood ratio test statistic is uniquely determined as $\boldsymbol{T}$. Although we assume that the mean vector has a specified direction, it is occasionally difficult to decide this direction. Therefore the specified direction $\delta$ may be different from the true direction $\boldsymbol{\delta}_{1}$. Thus in this section we give some numerical examples regarding the power of the test in a simple situation. Concretely we set up several types of specified direction $\delta$ and true direction $\delta_{1}$ and calculate the power of the test for each $\delta$ and $\boldsymbol{\delta}_{1}$. We investigate how the power of the test varies when $\boldsymbol{\delta}$ departs from $\boldsymbol{\delta}_{1}$. Simultaneously we compare our methods O-S and T-S with the method of Wang and McDermott (1998) denoted by W-M.

Suppose $p=2, n=30$. Since the power of the test depends on the unknown $\boldsymbol{\Sigma}$, we specify a form of $\boldsymbol{\Sigma}$ as

$$
\boldsymbol{\Sigma}=\left(\begin{array}{cc}
1 & 0.6 \\
0.6 & 1
\end{array}\right)
$$

Then we set up three types of $\boldsymbol{\delta}$ as

$$
\boldsymbol{\delta}=(1,1)^{\prime},(1,0.5)^{\prime},(1,0)^{\prime} .
$$

For each $\delta$ we give the power of the test under the alternative hypothesis

$$
H_{1}: \lambda=\lambda_{1} \text { for } \lambda_{1}=0.3,0.6
$$


when

$$
\boldsymbol{\delta}_{1}=(0,1)^{\prime}, \quad(0.5,1)^{\prime},(1,1)^{\prime},(1,0.5)^{\prime},(1,0)^{\prime} .
$$

Tables 1,2 and 3 give the power of the test in these cases respectively. Furthermore Figures 1,2 and 3 show the variation of the power of the test for $\mathbf{O}-\mathbf{S}, \mathbf{T}-\mathbf{S}$ and $\mathbf{W}-\mathbf{M}$ in Tables 1,2 and 3 respectively. When $\delta=\delta_{1}$, O-S is more powerful than T-S and W-M. But as $\boldsymbol{\delta}$ departs from $\boldsymbol{\delta}_{1}$, the power of the test in $\mathbf{O}-\mathbf{S}$ and $\mathbf{T}-\mathbf{S}$ decreases. On the other hand the power in $\mathbf{W}-\mathbf{M}$ is comparatively uniform for all $\boldsymbol{\delta}_{1}$. Thus $\mathbf{W}-\mathbf{M}$ is more powerful than $\mathbf{O}-\mathbf{S}$ and $\mathbf{T}-\mathbf{S}$ as $\boldsymbol{\delta}$ departs from $\boldsymbol{\delta}_{1}$.

Table 1: The power of the test under $H_{1}: \lambda=\lambda_{1}$ when $\delta=(1,1)^{\prime}$

\begin{tabular}{c|c|ccccc}
\hline & $\boldsymbol{\delta}_{1}$ & $(0,1)^{\prime}$ & $(0.5,1)^{\prime}$ & $(1,1)^{\prime}$ & $(1,0.5)^{\prime}$ & $(1,0)^{\prime}$ \\
\hline \multirow{3}{*}{$\lambda_{1}=0.3$} & $\mathbf{O}-\mathbf{S}$ & 0.166 & 0.290 & 0.450 & 0.290 & 0.166 \\
& T-S & 0.100 & 0.196 & 0.335 & 0.196 & 0.100 \\
& W-M & 0.398 & 0.299 & 0.359 & 0.299 & 0.398 \\
\hline \multirow{3}{*}{$\lambda_{1}=0.6$} & O-S & 0.376 & 0.748 & 0.941 & 0.748 & 0.376 \\
& T-S & 0.255 & 0.632 & 0.897 & 0.632 & 0.255 \\
& W-M & 0.943 & 0.829 & 0.897 & 0.829 & 0.943 \\
\hline
\end{tabular}
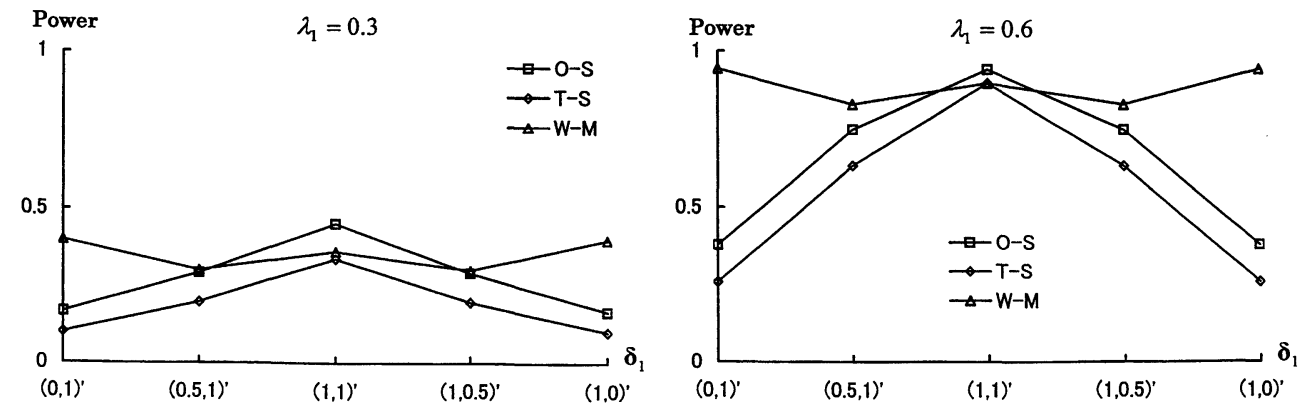

Fig. 1: Variation of power when $\delta=(1,1)^{\prime}$

Table 2: The power of the test under $H_{1}: \lambda=\lambda_{1}$ when $\boldsymbol{\delta}=(1,0.5)^{\prime}$

\begin{tabular}{c|c|ccccc}
\hline & $\boldsymbol{\delta}_{1}$ & $(0,1)^{\prime}$ & $(0.5,1)^{\prime}$ & $(1,1)^{\prime}$ & $(1,0.5)^{\prime}$ & $(1,0)^{\prime}$ \\
\hline \multirow{3}{*}{$\lambda_{1}=0.3$} & $\mathbf{O}-\mathbf{S}$ & 0.021 & 0.100 & 0.315 & 0.359 & 0.411 \\
& T-S & 0.043 & 0.064 & 0.229 & 0.271 & 0.313 \\
& $\mathbf{W}-\mathbf{M}$ & 0.398 & 0.299 & 0.359 & 0.299 & 0.398 \\
\hline \multirow{3}{*}{$\lambda_{1}=0.6$} & $\mathbf{O}-\mathbf{S}$ & 0.025 & 0.232 & 0.805 & 0.880 & 0.896 \\
& T-S & 0.056 & 0.149 & 0.708 & 0.821 & 0.837 \\
& W-M & 0.943 & 0.829 & 0.897 & 0.829 & 0.943 \\
\hline
\end{tabular}


Likelihood Ratio Test for Mean Vector with Specified Direction
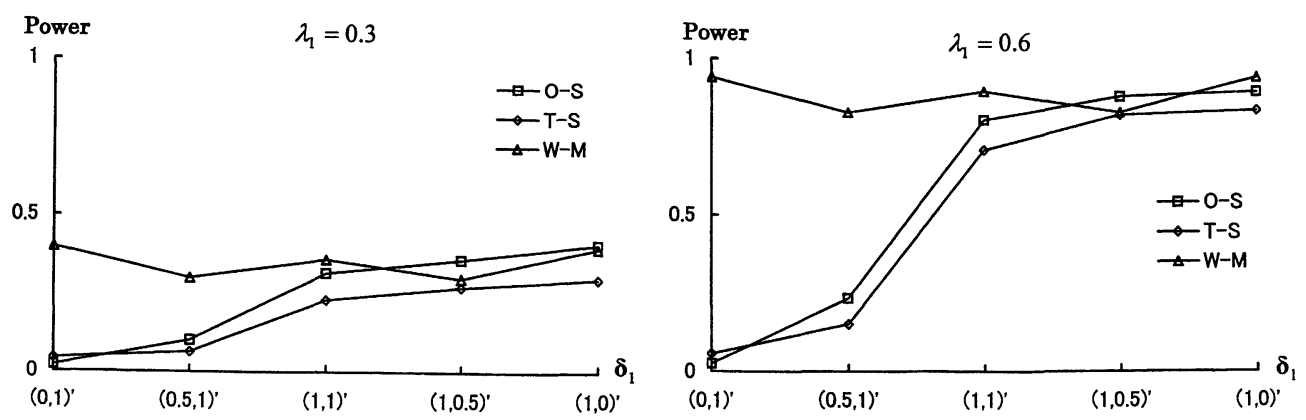

Fig. 2: Variation of power when $\delta=(1,0.5)^{\prime}$

Table 3: The power of the test under $H_{1}: \lambda=\lambda_{1}$ when $\delta=(1,0)^{\prime}$

\begin{tabular}{c|c|ccccc}
\hline & $\boldsymbol{\delta}_{1}$ & $(0,1)^{\prime}$ & $(0.5,1)^{\prime}$ & $(1,1)^{\prime}$ & $(1,0.5)^{\prime}$ & $(1,0)^{\prime}$ \\
\hline \multirow{3}{*}{$\lambda_{1}=0.3$} & O-S & 0.002 & 0.023 & 0.146 & 0.312 & 0.533 \\
& T-S & 0.154 & 0.037 & 0.086 & 0.211 & 0.410 \\
& W-M & 0.398 & 0.299 & 0.359 & 0.299 & 0.398 \\
\hline \multirow{3}{*}{$\lambda_{1}=0.6$} & O-S & 0.005 & 0.028 & 0.337 & 0.790 & 0.972 \\
& T-S & 0.449 & 0.046 & 0.221 & 0.678 & 0.949 \\
& W-M & 0.943 & 0.829 & 0.897 & 0.829 & 0.943 \\
\hline
\end{tabular}
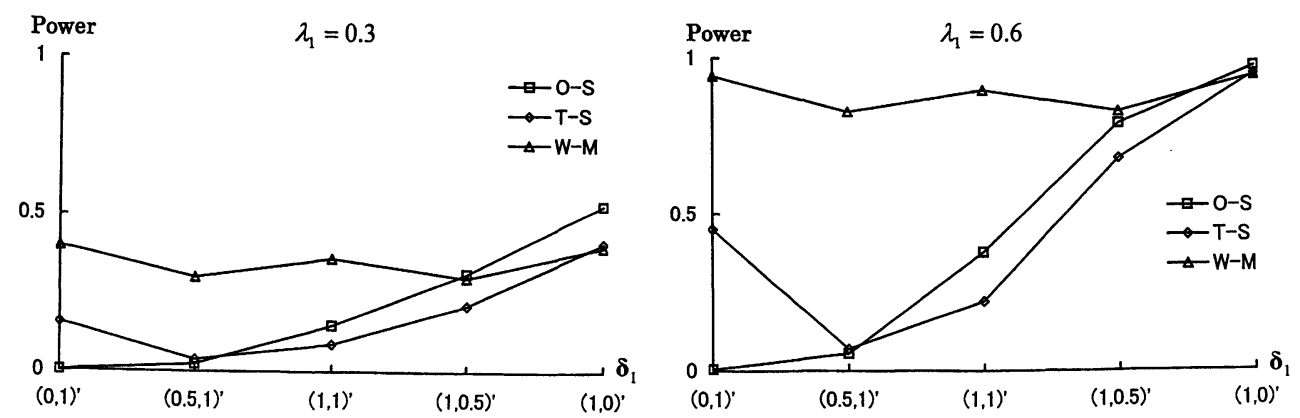

Fig. 3: Variation of power when $\delta=(1,0)^{\prime}$

\section{Conclusion}

In this study we discussed a one-sided test and a two-sided test based on multivariate normal response with unknown covariance matrix. Originally in a multivariate one-sided test we assume that the mean vector consists of non-negative components and in a multivariate two-sided test we assume that each component of the mean vector is simultaneously non-negative or non-positive and we test whether the vector is equal to zero or not. Although many authors considered the likelihood ratio test for these composite hypotheses, the procedures have theoretical complications. Thus we assumed that the difference between two mean vectors has a specified direction so that we could reduce the complications. Tang el al. (1989) derived the likelihood ratio test for the one-sided test under this assumption when the covariance matrix is known. In this study we constructed the likelihood ratio tests for 
the one-sided test and the two-sided test when the covariance matrix is unknown. Although the explicit formula of distribution of the likelihood ratio test statistic $\boldsymbol{T}$ does not exist, we constructed the acceptance region and the rejection region based on $\boldsymbol{T}$ and determined the critical value and the power of the test by using the multiple integration on these regions. We could determine the critical value independently of the unknown covariance matrix by using the conditional distribution considered by Wang and McDermott (1998).

But there exist some problems in our procedures. Although we assume that the mean vector has a specified direction, it is occasionally difficult to decide this direction. Thus the specified direction $\delta$ may be different from the true direction $\boldsymbol{\delta}_{1}$. From the results obtained by the numerical example we confirm that the method of Wang and McDermott (1998) is more powerful than O-S and T-S as $\boldsymbol{\delta}$ departs from $\boldsymbol{\delta}_{1}$. Furthermore, since we don't have the explicit formula of the distribution of the likelihood ratio test statistic $\boldsymbol{T}$, we must calculate the $p$-multiple integration to determine the critical value and the power of the test. If $p$ is large, the computation is complicated and takes a long time. Furthermore the power of the test depends on the unknown $\boldsymbol{\Sigma}$, although we could determine the critical value independently of $\boldsymbol{\Sigma}$ by using the conditional distribution considered by Wang and McDermott (1998). Therefore we should derive the null distribution of $\boldsymbol{T}$ which is independent of unknown $\boldsymbol{\Sigma}$.

\section{Acknowledgements}

The authors wish to thank the editor and the referees for valuable comments and suggestions.

\section{REFERENCES}

Anderson, T. W. (1984). An Introduction to Multivariate Statistical Analysis, 2nd edition. New York: Wiley.

Imada, T. and Douke, H. (2000). Two-sided test for bivariate normal mean vectors. Proceedings The Seventh Japan-China Symposium on Statistics, 349-352.

Inada, K. (1978a). Some bivariate tests of composite hypotheses with restricted alternatives. Reports of the Faculty of Science, Kagoshima University 11, 25-31.

Inada, K. (1978b). Some bivariate normal tests of composite hypotheses. Reports of the Faculty of Science, Kagoshima University 11, 33-36.

Kudo, A. (1963). A multivariate analogue of the one-sided test. Biometrika 50, 403-418.

Kudo, A. and Fujisawa, H. (1964). A bivariate normal test with two sided alternative. Memoirs of the Faculty of Science, Kyushu University Ser.A 18, 104-108.

Perlman, M. D. (1969). One-sided testing problems in multivariate analysis. The Annals of Mathematical Statistics 40, 549-567.

Sasabuchi, S. (1980). A test of a multivariate normal mean with composite hypotheses determined by linear inequalities. Biometrika 67, 429-439.

Sasabuchi, S. (1988). A multivariate one-sided test with composite hypotheses when the covariance matrix is completely unknown. Memoirs of the Faculty of Science, Kyushu University Ser.A 42, 37-46.

Seber, G. A. F. (1977). Linear Regression Analysis. New York: Wiley.

Tang, D.-I., Gnecco, C. and Geller, N. L. (1989). Design of group sequential clinical trials with multiple endpoints. Journal of the American Statistical Association 84, 776-779.

Wang, Y. and McDermott, M. P. (1998). Conditional likelihood ratio test for a nonnegative normal mean vector. Journal of the American Statistical Association 93, 380-386.

(Received February 2002, Accepted October 2002) 